Pacific Journal of Mathematic 


\title{
BIHOLOMORPHIC APPROXIMATION OF PLANAR DOMAINS
}

\author{
Bryan E. CaIn and Richard J. TondRa
}

This paper establishes the existence of a domain (open connected subset) $B$ of the complex plane $C$ such that for every domain $\Omega \subset C$ and every compact set $K \subset \Omega$, there is a biholomorphic embedding $e: B \rightarrow \Omega$, such that $K \subset e(B) \subset$ $\operatorname{cl}[e(B)] \subset \Omega$.

1. Introduction. Let $\Omega_{1}$ and $\Omega_{2}$ be domains (i.e., open connected sets) in the complex plane $C$ such that $\operatorname{cl} \Omega_{1} \subset \Omega_{2}$ (cl= closure). A domain $\Omega$ is a biholomorphic approximation of $\Omega_{1}$ with respect to $\Omega_{2}$ provided that there exists an invertible holomorphic function $e$ defined on $\Omega$ such that

$$
\operatorname{cl} \Omega_{1} \subset e(\Omega) \subset \operatorname{cl}[e(\Omega)] \subset \Omega_{2} .
$$

The mapping $e$ is a biholomorphic embedding (bh-embedding) of $\Omega$ into $\Omega_{2}$. ( $\Omega$ may also be considered a biholomorphic approximation of $\Omega_{2}$ with respect to $\Omega_{1}$.)

Homeomorphic domains may, of course, be biholomorphically inequivalent, and, moreover, may not even be close biholomorphic approximations of each other. For example, let $A(r, s)=\{z \in C: r<$ $|z|<s\}$ when $0<r<s<\infty$. Suppose that $0<\varepsilon<1<t<\infty$ and that $e$ is a $b h$-embedding of $A=A(r, s)$ such that

$$
\operatorname{cl} A(1, t) \subset e(A) \subset \operatorname{cl}[e(A)] \subset A(1-\varepsilon, t+\varepsilon) \text {. }
$$

By taking the modules of these ring domains (cf. [1]) we obtain the inequality $t<s / r<(t+\varepsilon) /(1-\varepsilon)$ which is precisely the condition $r$ and $s$ must satisfy for such an embedding $e$ to exist.

Our main result establishes the existence of a domain $B \subset C$ which is a biholomorphic approximation of every bounded domain $\Omega_{1}$ with respect to every domain $\Omega_{2}$ containing $\operatorname{cl} \Omega_{1}$.

2. The main theorem. Let $\hat{C}$ denote the Riemann sphere.

THEOREM 2.1. There exists a domain $B \subset C$ such that for every domain $\Omega \subset \hat{\boldsymbol{C}}$ and for every compact set $K \subset \Omega$ other than $\hat{\boldsymbol{C}}$ there exists a biholomorphic embedding $e: B \rightarrow \Omega$ such that $K \subset e(B) \subset$ $\operatorname{cl}[e(B)] \subset \Omega$.

REMARK. Actually such an embedding will exist if $\Omega$ is any connected Riemann surface (without boundary) and $K \subset \Omega$ is any planar compact surface other than $\hat{\boldsymbol{C}}$. ("Planar" means homeomorphic 
to a subset of $\hat{\boldsymbol{C}}$.) Indeed, by the trianguability of $\Omega$ there must exist a planar domain $\Omega_{0}$ such that $K \subset \Omega_{0} \subset \Omega$, and so it suffices to consider the planar case.

The following theorems are corollaries of Theorem 2.1.

COROLLARY 2.2. Let $K \neq \hat{\boldsymbol{C}}$ be a compact connected subset of a domain $\Omega \subset \hat{\boldsymbol{C}}$. Then $K=\bigcap_{i=1}^{\infty} B_{i}$ where each $B_{i}$ is bh-equivalent to $B$ and $\mathrm{cl} B_{i+1} \subset B_{i}$ for $i=1,2, \cdots$.

Corollary 2.3. Let $\Omega \neq \phi$ be a domain in $C$. Then $\Omega=\bigcup_{i=1}^{\infty} B_{i}$ where each $B_{i}$ is bh-equivalent to $B$ and $\operatorname{cl} B_{i} \subset B_{i+1}$ for $i=1,2, \cdots$.

3. Proofs. For each $a \in C$ and $r>0$ set $D(a, r)=\{z:|z-a|<r\}$ and let $\bar{D}(a, r)$ denote $\operatorname{cl} D(a, r)$. Set $D=D(0,1)$. A circle $\{z:|z-a|=r\}$ will be called "rational" provided that $\operatorname{Re} a, \operatorname{Im} a$, and $r>0$ are rational numbers. The topological boundary of a domain $\Omega$ will be denoted $\partial \Omega$.

To construct $B$ consider the domains $\Omega$ satisfying: (1) $\partial \Omega$ has finitely many components, (2) each component of $\partial \Omega$ is a rational circle, (3) cl $\Omega \subset D$ and its outer boundary is centered at the origin. Let $E_{1}, E_{2}, \cdots$ be an enumeration of these domains. Let $s_{j}$ be the radius of the outer boundary of $E_{j}$ and let $\phi_{j}$ be the linear fractional transformation of $D$ onto $H=\{z: \operatorname{Re} z>0\}$ which carries -1 to $0,+1$ to $\infty$, and $-s_{j}$ to 1 if $j=1$ and to $\phi_{j-1}\left(s_{j-1}\right)$ if $j>1$. Let $B=$ $H \backslash \bigcup_{j=1}^{\infty} \phi_{j}\left[D\left(0, s_{j}\right) \backslash E_{j}\right]$.

To show that $B$ has the desired properties, we prove the following lemma using the "small mesh grid" technique (often employed in texts on function theory), rather than the theory of trianguability. A bounded domain $\Omega \subset C$ will be called a Jordan domain if $\partial \Omega$ consists of finitely many disjoint Jordan curves.

LEMMA 3.1. Let $K$ be a compact subset of a domain $\Omega \subset C$. Then there exists a Jordan domain $\Omega_{0}$ such that $K \subset \Omega_{0} \subset \operatorname{cl} \Omega_{0} \subset \Omega$.

Sketch of proof. Since $\Omega$ is connected, there exists a connected compact set $K_{0}$ such that $K \subset K_{0} \subset \Omega$. Thus we may assume that $K$ is connected. With $r$ picked so small that $[K+\bar{D}(0, \sqrt{2} r)] \subset \Omega$ let $L$ be the union of those squares of a grid of squares with edge length $r$ which intersect $K$. If $a \in L$ is a vertex of precisely two squares of $L$ select the positive number $s_{a}<r / 2$ to be so small that $\bar{D}\left(a, s_{a}\right) \subset \Omega$. Let $L_{0}$ denote the union of all the $\bar{D}\left(a, s_{a}\right)$ 's. Then straightforward arguments show that $\Omega_{0}=\operatorname{int}\left(L \cup L_{0}\right)$ is the desired Jordan domain.

Now let $\Omega$ and $K$ be as described in Theorem 2.1. Lemma 3.1 
provides a Jordan domain $\Omega_{0}$ such that $K \subset \Omega_{0} \subset \operatorname{cl} \Omega_{0} \subset \Omega$. According to Theorem 2 page 237 of [2] there is a $b h$-embedding $h$ of $\Omega_{0}$ into $D$ such that (1) the outer boundary of $h\left(\Omega_{0}\right)$ is $\partial D$ and (2) $\partial\left[h\left(\Omega_{0}\right)\right]$ has finitely many components and each is a circle. Each of the circles bounding $h\left(\Omega_{0}\right)$ can be "approximated" arbitrarily closely by a rational circle which lies in $h\left(\Omega_{0}\right)$. We require that the approximation to the unit circle be centered at 0 . Since $h(K)$ is a compact subset of $h\left(\Omega_{0}\right)$, when the approximations are close enough, the approximating circles will bound a domain which contains $h(K)$. This region, by its definition, is one of the $E_{j}$ 's, say $E_{k}$. Then

$$
h(K) \subset E_{k} \subset \phi_{k}^{-1}(B) \subset h\left(\Omega_{0}\right)
$$

and so applying $h^{-1}$ will establish Theorem 2.1.

To prove Corollary 2.2 we let $B_{1}=e_{1}(B)$ where $e_{1}$ is the $b h$ embedding of $B$ such that $K \subset B_{1} \subset \operatorname{cl} B_{1} \subset \Omega$. For $i>1$ we let $G_{i}$ be the component of $[K+D(0,1 /(i-1))] \cap B_{i-1}$ which contains $K$, and we set $B_{i}=e_{i}(B)$ where $e_{i}$ is the $b h$-embedding of $B$, given by Theorem 2.1, such that $K \subset B_{i} \subset \operatorname{cl} B_{i} \subset G_{i}$.

To prove Corollary 2.3 we pick $a \in \Omega$ and for large $n$ we can let $G_{n}$ be the component of $\{z: \operatorname{dist}(z, C \backslash \Omega)>1 / n$ and $|z|<n\}$ which contains $a$. Since $\mathrm{cl} G_{n}$ is a compact subset of $G_{n+1}$ there exists a bh-embedding $e_{n}: B \rightarrow G_{n+1}$ such that $B_{n}=e_{n}(B) \supset \operatorname{cl} G_{n}$. That $\Omega=$ U $G_{n}$ (and hence $\Omega=\bigcup B_{n}$ ) follows from the arc connectedness of $\Omega$. These $B_{n}$ 's are the required domains (except for re-indexing).

4. Some applications to holomorphic extension problems. Let $K \subset C$ be compact and let $f: K \rightarrow C$. It is easy to extend $f$ to a holomorphic function $F$ defined on a domain containing $K$ (caution: domains are connected) if there exist: (1) a domain $\Omega$, (2) a biholomorphic function $e$ on $\Omega$ such that $K \subset e(\Omega)$, and (3) a holomorphic extension $G$ of $g=\left.f \circ e\right|_{e^{-1}(K)}$ to all of $\Omega$. Indeed $F=G \circ e^{-1}$ is the required extension. Conversely if $f$ has such an extension $F$ the existence of $\Omega, e$, and $G$ is trivial. For let the domain $\Omega$ be the domain of $F$, set $e(z)=z$, and take $G=F$. Thus we have an equivalent formulation of the problem of holomorphically extending a function $f: K \rightarrow C$ to a domain containing $K$. Theorem 4.2 shows that another equivalent formulation is obtained when in the discussion above the variable domain $\Omega$ is replaced by the fixed domain $B$. We first show that for a more restricted class of sets $K$ this extension question is very naturally formulated with $D$ in the role of $\Omega$.

TheOREM 4.1. Let $K \subset \boldsymbol{C}$ be compact and let $f: K \rightarrow C$. Suppose 
that $K$ and $C \backslash K$ are connected. Then there exists a holomorphic extension $F$ of $f$ to a domain containing $K$ if and only if there exist (a) a bh-embedding $e$ of $D$ such that $K \subset e(D)$ and (b) a holomorphic extension $G$ of $g=\left.f \circ e\right|_{e^{-1}(K)}$ to all of $D$.

Proof. Since the "if" part of this theorem is treated in the discussion above we confine our remarks to the "only if" part. Assume that the extension $F$ exists, and let $\Omega \supset K$ be its domain. It suffices to find a bh-mapping $e$ of $D$ such that $K \subset e(D) \subset \Omega$. This is trivial if $K$ is a singleton: so we assume $K$ is not a singleton. Then the Riemann Mapping theorem shows that $\hat{\boldsymbol{C}} \mid K$ is bh-equivalent to $D$ (it is simply connected because $K$ is connected). Let $h: \widehat{\boldsymbol{C}} \backslash K \rightarrow D$ be the Riemann mapping. Since $h^{-1}(\bar{D}(0, r))$ is simply connected for $0<r<1$ we know that $V_{r}=\hat{\boldsymbol{C}} \backslash h^{-1}(\bar{D}(0, r))$ is nonempty, open, and simply connected for $0<r<1$. Thus each $V_{r}$ with $0<r<1$ is $b h$-equivalent to $D$. Since $h(\hat{C} \mid \Omega)$ is a compact subset of $D$ it lies in $D(0, s)$ for some $s<1$, and the Riemann mapping $e$ of $D$ onto $V_{s}$ is the required map.

If in Theorem 4.1 $D$ is replaced by $B$ the assumption that $K$ and $C \backslash K$ are connected may be dropped.

THEOREM 4.2. Let $K \subset C$ be compact and let $f: K \rightarrow C$. There exists a holomorphic extension $F$ of $f$ to a domain containing $K$ if and only if there exist (a) a bh-mapping $e$ of $B$ such that $K \subset e(B)$ and (b) a holomorphic extension $G$ of $g=\left.f \circ e\right|_{e^{-1}(K)}$ to all of $B$.

Proof. As in the proof of Theorem 4.1 the "if" part has already been settled and we begin the "only if" part by letting $\Omega \supset K$ be the domain of $F$. An application of Theorem 2.1 gives a $b h$-embedding $e$ of $B$ such that $K \subset e(B) \subset \Omega$. This is the required mapping.

REMARK. Comparing Theorems 4.1 and 4.2 tempts one to conjecture the existence of a sequence of domains $D=\Omega_{1}, \Omega_{2}, \cdots, \Omega_{\infty}=B$ such that $\hat{\boldsymbol{C}} \backslash \Omega_{n}$ has $n$ components and for which Theorem 4.1 will remain true when it is modified by: (1) Replacing its second sentence with "Suppose $K$ is connected and $C \backslash K$ has $n$ components", and (2) Replacing $D$ with $\Omega_{n}$. The discussion in the introduction shows that this conjecture fails, since for $n=2, \Omega_{2}$ must be $b h$-equivalent to $A(r, s)$ for some $r, s$ with $0 \leqq r<s \leqq \infty$ and so $\Omega_{2}$ cannot be embedded between $A(1, t)$ (the domain of $f$ ) and $A(1-\varepsilon, t+\varepsilon)$ (the domain of the extension $F)$ unless $t<s / r<(t+\varepsilon) /(1+\varepsilon)$. 


\section{REFERENCES}

1. W.H.J. Fuchs, Topics in the Theory of Functions of One Complex Variable, Van Nostrand Mathematical Studies $\$ 12$, D. Van Nostrand, Princeton, N. J., 1967.

2. G. M. Goluzin, Geometric Theory of Function of a Complex Variable, Translations of Math. Mono., vol. 26, Providence, Rhode Island, 1969.

Received July 19, 1973.

Iowa State University 



\section{PACIFIC JOURNAL OF MATHEMATICS}

EDITORS

RICHARD ARens (Managing Editor)

University of California

Los Angeles, California 90024

\section{R. A. Beaumont}

University of Washington

Seattle. Washington 98105

\section{J. DugundjI}

Department of Mathematics University of Southern California Los Angeles, California 90007

D. Gilbarg and J. Milgram

Stanford University

Stanford. California 94305

\section{ASSOCIATE EDITORS}
E. F. BECKENBACH
B. H. NEUMANN
F. WOLF
K. Yoshida

\section{SUPPORTING INSTITUTIONS}

\author{
UNIVERSITY OF BRITISH COLUMBIA \\ CALIFORNIA INSTITUTE OF TECHNOLOGY \\ UNIVERSITY OF CALIFORNIA \\ MONTANA STATE UNIVERSITY \\ UNIVERSITY OF NEVADA \\ NEW MEXICO STATE UNIVERSITY \\ OREGON STATE UNIVERSITY \\ UNIVERSITY OF OREGON \\ OSAKA UNIVERSITY
}

\author{
UNIVERSITY OF SOUTHERN CALIFORNIA \\ STANFORD UNIVERSITY \\ UNIVERSITY OF TOKYO \\ UNIVERSITY OF UTAH \\ WASHINGTON STATE UNIVERSITY \\ UNIVERSITY OF WASHINGTON

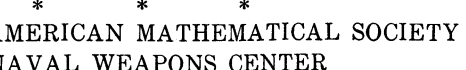

The Supporting Institutions listed above contribute to the cost of publication of this Journal, but they are not owners or publishers and have no responsibility for its content or policies.

Mathematical papers intended for publication in the Pacific Journal of Mathematics should be in typed form or offset-reproduced, (not dittoed), double spaced with large margins. Underline Greek letters in red, German in green, and script in blue. The first paragraph or two must be capable of being used separately as a synopsis of the entire paper. Items of the bibliography should not be cited there unless absolutely necessary, in which case they must be identified by author and Journal, rather than by item number. Manuscripts, in duplicate if possible, may be sent to any one of the four editors. Please classify according to the scheme of Math. Rev. Index to Vol. 39. All other communications to the editors should be addressed to the managing editor, or Elaine Barth, University of California, Los Angeles, California, 90024.

100 reprints are provided free for each article, only if page charges have been substantially paid Additional copies may be obtained at cost in multiples of 50 .

The Pacific of Journal Mathematics is issued monthly as of January 1966. Regular subscription rate: $\$ 72.00$ a year (6 Vols., 12 issues). Special rate: $\$ 36.00$ a year to individual members of supporting institutions.

Subscriptions, orders for back numbers, and changes of address should be sent to Pacific Journal of Mathematics, 103 Highland Boulevard, Berkeley, California, 94708.

\section{PUBLISHED BY PACIFIC JOURNAL OF MATHEMATICS, A NON-PROFIT CORPORATION}

Printed at Kokusai Bunken Insatsusha (International Academic Printing Co., Ltd.), 270, 3-chome Totsuka-cho. Shinjuku-ku, Tokyo 160. Japan.

Copyright (C) 1973 by Pacific Journal of Mathematics Manufactured and first issued in Japan 


\section{Pacific Journal of Mathematics}

\section{Vol. 52, No. $2 \quad$ February, 1974}

Harm Bart, Spectral properties of locally holomorphic vector-valued functions .....

J. Adrian (John) Bondy and Robert Louis Hemminger, Reconstructing infinite

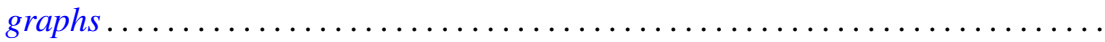

Bryan Edmund Cain and Richard J. Tondra, Biholomorphic approximation of planar

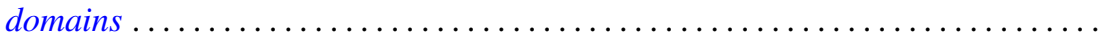

Richard Carey and Joel David Pincus, Eigenvalues of seminormal operators,

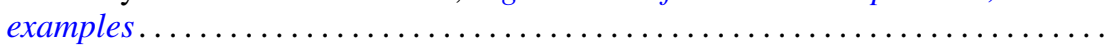

Tyrone Duncan, Absolute continuity for abstract Wiener spaces . . . . . . . . . . . . Joe Wayne Fisher and Louis Halle Rowen, An embedding of semiprime

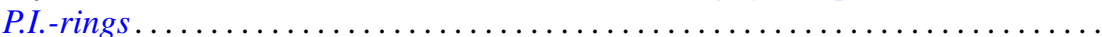

Andrew S. Geue, Precompact and collectively semi-precompact sets of semi-precompact continuous linear operators. . . . . . . . . . . . . . . .

Charles Lemuel Hagopian, Locally homeomorphic $\lambda$ connected plane continua ..... . Darald Joe Hartfiel, A study of convex sets of stochastic matrices induced by

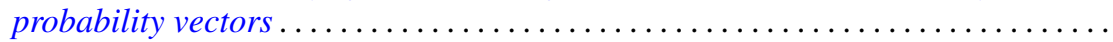

Yasunori Ishibashi, Some remarks on high order derivations $\ldots \ldots \ldots \ldots \ldots \ldots \ldots$ Donald Gordon James, Orthogonal groups of dyadic unimodular quadratic forms.

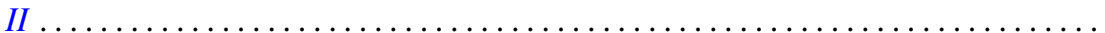

Geoffrey Thomas Jones, Projective pseudo-complemented semilattices . . . . . . . . . Darrell Conley Kent, Kelly Denis McKennon, G. Richardson and M. Schroder,

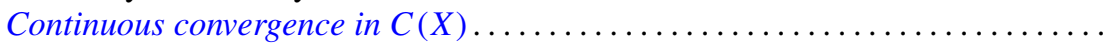

J. J. Koliha, Some convergence theorems in Banach algebras ...

Tsang Hai Kuo, Projections in the spaces of bounded linear oper

George Berry Leeman, Jr., A local estimate for typically real functions . .

475

Andrew Guy Markoe, A characterization of normal analytic spaces by the

homological codimension of the structure sheaf .........

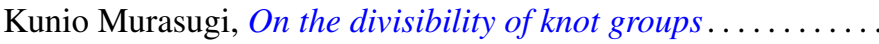

John Phillips, Perturbations of type I von Neumann algebras.

Billy E. Rhoades, Commutants of some quasi-Hausdorff matrices . .

David W. Roeder, Category theory applied to Pontryagin duality

Maxwell Alexander Rosenlicht, The nonminimality of the differential closure .

Peter Michael Rosenthal, On an inversion theorem for the general Mehler-Fock transform pair.

Alan Saleski, Stopping times for Bernoulli automorphisms

John Herman Scheuneman, Fundamental groups of compact complete locally affine complex surfaces. II. ........................

Vashishtha Narayan Singh, Reproducing kernels and operators with a cyclic vector. I. .

Peggy Strait, On the maximum and minimum of partial sums of random variables.

J. L. Brenner, Maximal ideals in the near ring of polynomials modulo 2 .

Ernst Gabor Straus, Remark on the preceding paper: "Ideals in near rings of polynomials over a field" ..........................

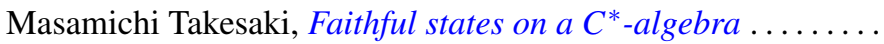

R. Michael Tanner, Some content maximizing properties of the regular simplex.

Andrew Bao-hwa Wang, An analogue of the Paley-Wiener theorem for certain

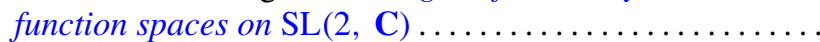

